\title{
LUCES Y SOMBRAS DE LA POLÍTICA DE LA COMPETENCIA EN ITALIA
}

\author{
Sergio A. Berumen \\ Universidad Rey Juan Carlos (Madrid, España) \\ sergio.berumen@urjc.es
}

\section{Resumen}

En el pasado las autoridades responsables del gobierno italiano se limitaron a reaccionar ante las presiones que ejercía la Comisión Europea en materia de la competencia, pero salvo muy escasas excepciones los distintos gobiernos de este país han actuado de forma reactiva. En este artículo se muestra que la política de la competencia italiana ha sido errática: en ocasiones la autoridad actuó oportunamente, pero ha habido otras en las que resultaba urgente su intervención, y esta se hizo esperar. Para el efecto, el interés de este artículo se centra en analizar la evolución del mercado y en señalar los avances más significativos que se han alcanzado en materia de liberalización del mercado interno.

Palabras claves: Economía italiana, política de la competencia, regulación, servicios públicos, sector telecomunicaciones.

\begin{abstract}
In the past the responsible authorities of the Italian government have limited their performance to react to those pressures coming from the European Commission in respect to competence matters, but only in some exceptional circumstances the different Italian governments have acted in a reactive way. In this article it is demonstrated that the Italian policies regarding competence have been erratic; sometimes the authorities have reacted in time, but in some other occasions their intervention was quite urgent and it has arrived later than it was expected. To this effect, interest is focused in analyzing the evolution of the market and in pointing out the most significative advances that have been reached in respect to their internal market liberalization.
\end{abstract}

Key words: Italian economy, competition policy, telecommunications, regulation, competition, services. 


\section{Introducción}

Según el Index 2008, elaborado por la Heritage Foundation, la economía italiana es considerada "libre" en $62,5 \%$, lo que la sitúa en la posición 64 entre las economías más libres del mundo. Esto significa que ha bajado 0,2 puntos respecto al 2007, lo que representa que no se está consiguiendo el mismo grado de avance que el logrado por otros países que se han dado a la tarea de realizar reformas más profundas. Italia alcanza buenos resultados en materia de libertad de negocio, comercio e inversiones y libertades laborales, pero está por debajo del promedio mundial en cuanto a la libertad fiscal, lo que se desprende del inmenso tamaño del Estado. La mano visible del Estado tradicionalmente ha desempeñado un papel importante en la economía italiana por su intervención directa en los sectores productivos (v. g. la vasta cantidad de empresas públicas). Para Stefano Micossi ${ }^{1}$, esta cuestión es un lamentable legado del régimen fascista.

Antiguamente el Estado garantizaba concesiones y subvenciones a las empresas públicas, lo que las convertía a todos los efectos en monopolios. El impacto de este proceder se reflejó en que los precios no se regulaban conforme la ley de la oferta y la demanda (por ejemplo, los precios del transporte público y del agua eran muy bajos, mientras que los de la electricidad, del gas natural y de las telecomunicaciones eran desorbitados), lo que se tradujo en un incremento constante del gasto público, pero también en una baja productividad de las empresas.

1. Director general de la Associazione fra le Società per Azione (Assonime, Asociación entre la Sociedad para la Acción, de Roma) y profesor del College of Europe (Colegio de Europa, en Brujas).
Hoy en día varias organizaciones estatales se han vendido total o parcialmente a empresarios privados, si bien - como lo reflejan los indicadores de la regularización del mercado de la Organización para la Cooperación y el Desarrollo Económicos (OCDE, 2007) — todavía hay una potente intervención del Estado en comparación con otros países comunitarios (tanto en el control directo sobre determinadas empresas como a la cantidad de empresas públicas), en especial en el caso de los medios de comunicaciones, del transporte, de los servicios públicos y el de la energía. El cuadro 1 muestra el nivel de intervención que ejercía el Estado en las empresas italianas hasta el 2003.

\section{Liberalización del mercado interno y privatizaciones}

Desde el 2003 hasta el día de hoy ha habido avances en materia de intervención, aunque ciertamente el Estado italiano ha continuado practicando medidas de regulación en el mercado, principalmente a través del control que ejercen los ministerios, a la cabeza de los cuales está el Ministero dell' Economia e delle Finanze (MEF), y en menor medida a través de las decisiones emanadas de la Comisión Interministerial de Planificaciones Europeas (CIPE). Los sectores que mayoritariamente se han visto intervenidos en los últimos años son el ferroviario, el postal, el de la gestión de los recursos hidráulicos y el agrario, debido a que este país ocupa un lugar destacado en la repartición de los fondos de la PAC $^{2}$ (Política Agraria Común de las Comunidades Europeas). En los asuntos

2. Esto significa que 4 de cada 10 horas que trabajan los italianos lo hacen para mantener el aparato de gobierno. Véase el Index 2008, acerca de Italia. 


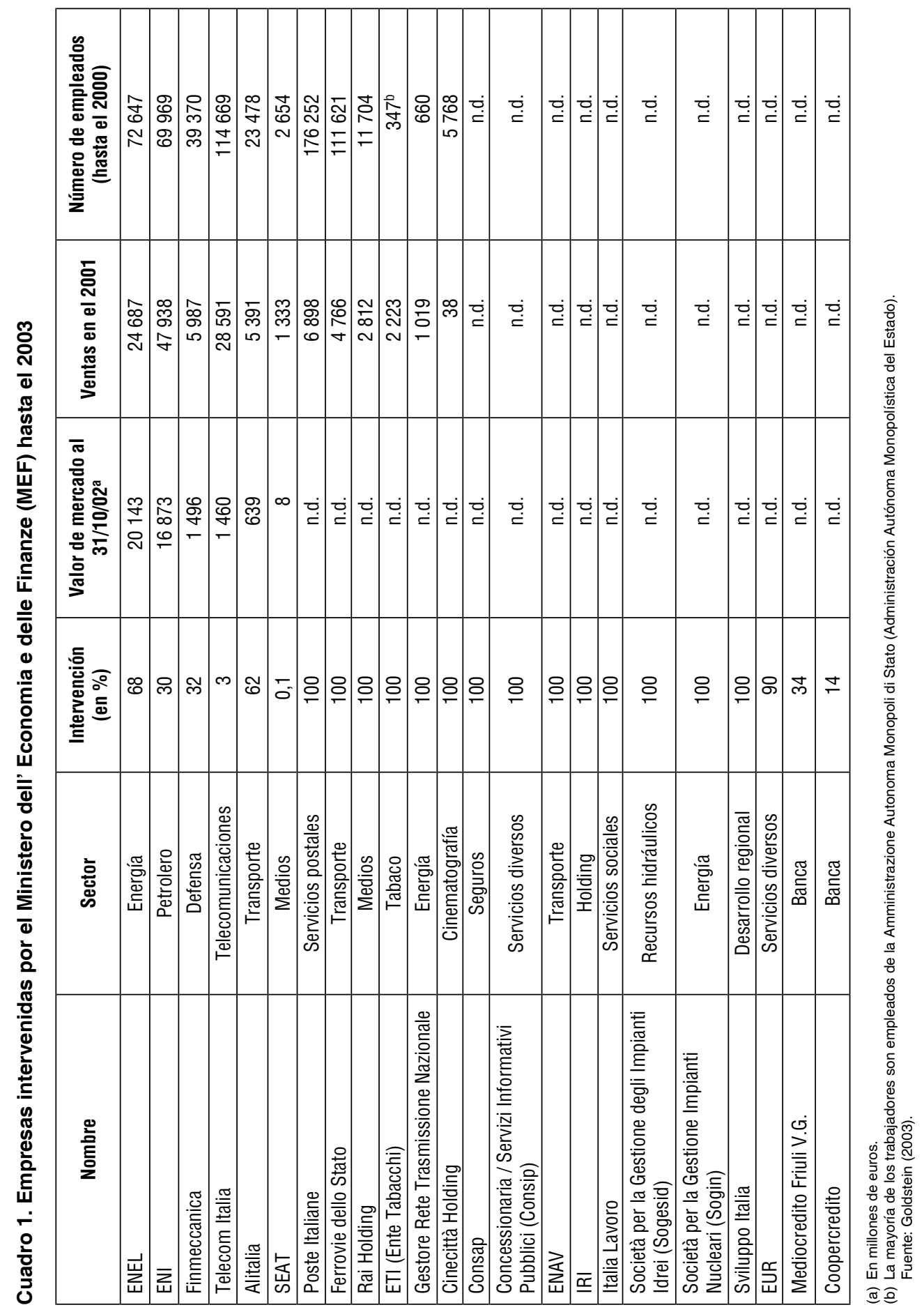


más graves el Estado ha intervenido en la fijación de precios, como es el caso del agua potable y la electricidad, del gas, de los peajes de las autopistas, de la venta de medicinas que requieren receta, de las telecomunicaciones y del tráfico de automóviles en las grandes ciudades. Todo este cúmulo de actividades ha propiciado que el Estado italiano haya desarrollado un tamaño desproporcionado, lo que se ha traducido en una gestión torpe y cara del aparato de gobierno. Como lo advierte Zimermann (2008), una muestra de ello es que su mantenimiento consume cerca del $40 \%$ de los impuestos que pagan los contribuyentes italianos.

El afán regulatorio en Italia es incluso superior al que prevalece en la mayoría de los países comunitarios. A modo de ejemplo, en el caso de la industria farmacéutica, en diversas ocasiones ha vencido la vigencia de las patentes a nivel mundial, pero en Italia de manera expedita se ha concedido una prolongación de esa vigencia. El excesivo intervencionismo tuvo su origen en la década de los setenta cuando los poderosos sindicatos presionaron a los gobiernos para que se promoviesen leyes que protegiesen los derechos laborales (como lo fueron los decretos que impedían los despidos masivos, lo que condujo a que los empresarios reaccionasen con renuencia a crear contratos indefinidos). Para la década de los ochenta los hábiles empresarios impidieron que los sindicatos penetrasen con fuerza en las empresas, pero fueron incapaces de atemperar la influencia que estos ejercieron sobre el gobierno. Para Micossi (2006) las condiciones de contratación impuestas por los sindicatos desde entonces han generado un lastre hasta nuestros días. Una muestra de ello está en que en la actualidad la determinación de los salarios sigue ignorando los resultados de las empresas y en general el desempeño de cada trabajador, lo que impacta sensiblemente en los niveles de productividad.

Aunado a todo lo anterior, en muchos casos la provisión de algunos servicios han permanecido en manos de los consistorios locales, los cuales no se han caracterizado por ser buenos gestores. Salvo honrosas excepciones, los gobiernos municipales han sido ineficientes cuando se trata de mejorar la calidad de los servicios que prestan y de reducir los costos, como queda patente en el caso de los procedimientos de concesión de licencias a nivel local, manifiestamente carentes de plena transparencia y con la eterna propensión a la corrupción.

El cuadro 2 muestra el desarrollo de la libertad económica en Italia entre 1980 y 2005. Según el Economic Freedom of the World (2007), en su informe mundial de libertad económica de 2007, si asignamos una escala entre 0 y 10 a cada categoría analizada (en que 0 es el peor y 10 el óptimo grado de libertad económica), los resultados más positivos se encuentran en:

i) reducción del tamaño del Estado (mejoró de 4,6 en el 2000 a 5,9 puntos en el 2005);

ii) inversión estatal (se han logrado notas sobresalientes a partir de 2003);

iii) reducción de subsidios;

iv) regulaciones restrictivas;

v) un aumento de la libertad en el sector financiero; $y$

vi) la reducción de los costos derivados de la gestión del aparato de gobierno.

Particularmente llama la atención el apartado respectivo al pago de dádivas, más comúnmente conocidas como "mordidas". 


\begin{tabular}{|c|c|c|c|c|c|c|c|c|c|c|c|c|c|c|c|c|c|c|}
\hline \multirow{2}{*}{ 옹 } & \multirow{2}{*}{ 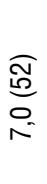 } & & $\begin{array}{l}\widehat{E} \\
\stackrel{\mathfrak{N}}{\mathfrak{N}}\end{array}$ & $\begin{array}{l}\text { o- } \\
\text { ఏ్ }\end{array}$ & $\begin{array}{l}\widehat{0} \\
\infty\end{array}$ & & & & & & & & & & & & & \\
\hline & & os & $\stackrel{\sim}{\sim}$ & $\stackrel{1}{\nabla}$ & $\begin{array}{l}\text { 응 } \\
\text { (1) }\end{array}$ & $\stackrel{\forall}{\sigma}$ & $\stackrel{m}{\forall}$ & $\hat{m}$ & $\begin{array}{l}\infty \\
0 \\
0^{-}\end{array}$ & $\hat{\sigma}$ & $\bar{\sigma}$ & $\stackrel{g}{\sim}$ & $\stackrel{\nabla}{\forall}$ & $\tilde{\sigma}^{\infty}$ & $\stackrel{\infty}{\sim}$ & o & 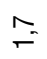 & $\stackrel{⿱}{N}$ \\
\hline \multirow{2}{*}{ 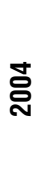 } & \multirow{2}{*}{$\begin{array}{l}\frac{\widehat{l}}{80} \\
\text { o } \\
6 \\
0\end{array}$} & & $\begin{array}{l}\widehat{\mathbb{v}} \\
\mathbb{d}\end{array}$ & $\begin{array}{l}\widehat{\infty} \\
\bar{\Xi}\end{array}$ & $\begin{array}{l}\widehat{\hat{\theta}} \\
\hat{\theta}\end{array}$ & & & & & & & & & & & & & \\
\hline & & $\mathscr{C}^{\circ}$ & $\stackrel{\bullet}{\sigma}$ & $\underset{\forall}{\sim}$ & $\begin{array}{l}\circ \\
\text { ○ }\end{array}$ & $\begin{array}{l}\infty \\
L^{\circ}\end{array}$ & $\underset{F}{\sim}$ & $\stackrel{\bullet}{\oplus}$ & $\mid$\begin{tabular}{|l}
$\mid 0$ \\
10 \\
10
\end{tabular} & 0 & $\stackrel{\widetilde{v}}{\tilde{\sigma}}$ & 足 & $\begin{array}{l}L^{\circ} \\
L^{\circ}\end{array}$ & $\tilde{\sigma}^{\circ}$ & $\hat{\sim}$ & $\hat{L}^{\circ}$ & $\stackrel{\infty}{\leftarrow}$ & $\stackrel{\llcorner}{\sim}$ \\
\hline \multirow{2}{*}{ ֻั } & \multirow{2}{*}{ 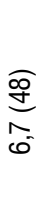 } & & $\begin{array}{l}\stackrel{J}{d} \\
\mathbb{E}\end{array}$ & $\begin{array}{l}\widehat{\alpha} \\
\bar{N}\end{array}$ & $\begin{array}{l}\widehat{E} \\
\hat{\theta}\end{array}$ & & & & & & & & & & & & & \\
\hline & & $\underset{\omega 0}{6}$ & $\stackrel{\mathscr{O}}{\forall}$ & $\underset{\forall}{\sim}$ & $\begin{array}{l}\circ \\
\text { ㅇ }\end{array}$ & $\mathscr{L}^{0}$ & $\stackrel{m}{\forall}$ & $\hat{m}$ & $\stackrel{\infty}{\forall}$ & 오 & $m^{m}$ & $L^{\circ}$ & $\stackrel{\mathscr{m}}{\dot{m}}$ & $\stackrel{\circ}{\circ}$ & $\tilde{\sim}$ & $\begin{array}{l}0 \\
\text { Li }^{\circ}\end{array}$ & $\stackrel{I}{=}$ & $\stackrel{N}{N}$ \\
\hline \multirow{2}{*}{ ণั } & \multirow{2}{*}{$\begin{array}{l}\text { ल্লু } \\
\text { o } \\
\text { N }\end{array}$} & & $\begin{array}{l}\widetilde{\widetilde{d}} \\
\tilde{\mathbb{d}}\end{array}$ & $\begin{array}{l}\widehat{N} \\
\text { d }\end{array}$ & $\begin{array}{l}\text { o } \\
\text { ô } \\
\text { a }\end{array}$ & & & & & & & & & & & & & \\
\hline & & $\stackrel{\curvearrowright}{\approx}$ & $\stackrel{\infty}{\forall}$ & $\stackrel{\bullet}{\forall}$ & $\begin{array}{l}\circ \\
0\end{array}$ & 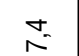 & $\sqrt{60}$ & $\stackrel{m}{n}$ & $\begin{array}{l}0 \\
0\end{array}$ & 응 & $m^{m}$ & $\stackrel{2}{\sim}$ & $\stackrel{0}{\infty}$ & $\stackrel{\circ}{\circ}$ & $\begin{array}{l}\circ \\
\aleph^{\prime}\end{array}$ & $\stackrel{\mathscr{O}}{\forall}$ & $\stackrel{m}{\sim}$ & 웅 \\
\hline \multirow{2}{*}{ 호 } & \multirow{2}{*}{ 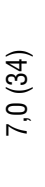 } & & $\begin{array}{l}0 \\
\text { d } \\
\text { d }\end{array}$ & 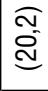 & & & & & & & & & & & & & & \\
\hline & & $\stackrel{0}{\forall}$ & $\hat{\sigma}$ & $\stackrel{0}{\forall}$ & O) & $\stackrel{\Xi}{\sim}$ & $\begin{array}{l}\text { os } \\
\text { L }\end{array}$ & $\begin{array}{l}0 \\
\forall\end{array}$ & $\begin{array}{l}m \\
c^{\prime}\end{array}$ & $\stackrel{\circ}{\circ}$ & $\overline{60}$ & \begin{tabular}{|l|}
$\infty$ \\
0 \\
0
\end{tabular} & $\stackrel{\mathscr{m}}{\oplus}$ & $\stackrel{\circ}{\circ}$ & $\stackrel{m}{\sim}$ & $\begin{array}{l}0 \\
10\end{array}$ & $\stackrel{\circ}{\sim}$ & $\begin{array}{l}\infty \\
0^{-}\end{array}$ \\
\hline \multirow{2}{*}{ ్ㅗ } & \multirow{2}{*}{$\begin{array}{l}\text { స్ర } \\
\text { ז }\end{array}$} & & 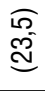 & 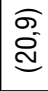 & & & & & & & & & & & & & & \\
\hline & & $\stackrel{\bullet}{\forall}$ & $\stackrel{\infty}{\sim}$ & $\stackrel{\nabla}{+}$ & \% & $\hat{N}$ & $\stackrel{\infty}{\underset{\sim}{*}}$ & $\widehat{T}$ & $\stackrel{\infty}{N}$ & $\begin{array}{l}\circ \\
\text { ○. }\end{array}$ & $\begin{array}{l}\text { "ח) } \\
10\end{array}$ & $\hat{\sigma}^{\prime}$ & 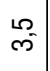 & $\stackrel{\circ}{\circ}$ & $\bar{\sim}$ & \begin{tabular}{|l}
$m$ \\
0 \\
0
\end{tabular} & $\begin{array}{l}\sigma \\
\sigma^{\circ}\end{array}$ & 芯 \\
\hline & 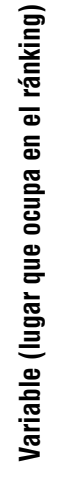 & 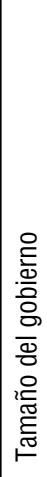 & 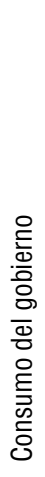 & $\begin{array}{l}\text { 음 } \\
\text { 응 } \\
\text { 을 }\end{array}$ & 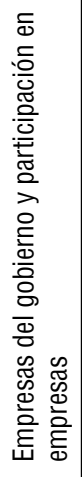 & 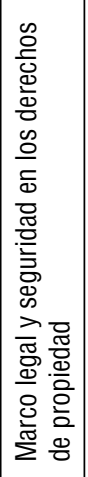 & 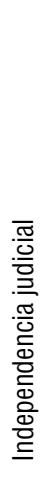 & 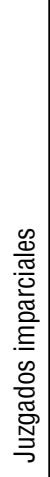 & 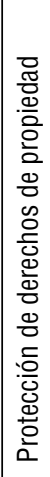 & 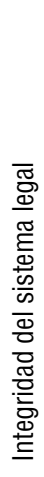 & 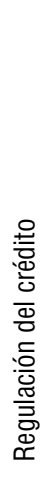 & 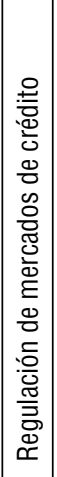 & 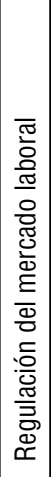 & 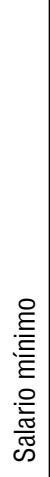 & 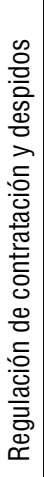 & 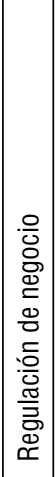 & 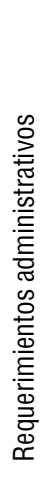 & 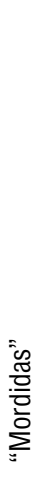 \\
\hline
\end{tabular}


En 1995, primer año en que se tomó el pulso a este indicador, Italia registró un lamentable 2,9; para el 2004 la cifra subió a 7,5, y un año después bajó una décima (al $7,4)$. Esto significa que el gobierno italiano ha hecho grandes avances en la lucha contra esta lacra social, pero aún dista de los 9,3 de Suecia o los 9,4 puntos que orgullosamente ostenta Finlandia.

Por otra parte, los resultados son francamente negativos en lo concerniente a:

i) regulación laboral;

ii) fijación de precios (los criterios se han mantenido inalterados durante los últimos 25 años); y

iii) barreras de entrada a empresas extranjeras.

Como se puede apreciar, en el cuadro 2 hemos resaltado aquellos indicadores que resultan más preocupantes. El apartado más inquietante es el referente a la independencia judicial, que ha mantenido una caída constante desde el 2002 hasta llegar a 4,3 , un dato malo sin paliativos. Lo mismo ocurre en el caso de la imparcialidad de los juzgados, con 3,7 puntos. En lo referente a la regulación a la que se enfrentan las empresas, los datos señalan una constante que apenas supera el aprobado, lo que no significa que sea un dato positivo. El indicador peor valorado es el referente a los requerimientos administrativos. En el 2000 tenía 6,9 puntos, pero desde entonces ha venido sufriendo un acusado descenso hasta alcanzar los 1,7 puntos en el 2005 . Este dato señala que el gobierno tiene que hacer denodados esfuerzos por simplificar los abrumadores trámites a los que se enfrentan los empresarios.

Pero sería injusto ignorar el empeño que los últimos gobiernos italianos han dedicado por hacer de su país un sitio más acorde con las reglas de la competencia. La muestra más notable de ello fue la creación en 1990 de la Autorità Garante de la Concorrenza e del Mercato ${ }^{3}$ (AGCM), organismo que se encarga de vigilar el buen funcionamiento de la competencia en el mercado. Adicionalmente - como lo señala Van Siclen (2000) - se establecieron instituciones especiales facultadas para regular y supervisar el correcto funcionamiento en los sectores de la electricidad, de la televisión y de las telecomunicaciones. La muestra más reciente del empeño del gobierno en crear un marco para liberalizar los mercados es el Decreto 223/206 (de 2006, también conocido como Decreto Bersani), que tiene la finalidad de reducir las barreras al comercio (especialmente de entrada) y de moderar el tamaño de la burocracia.

El denominado Decreto Bersani originalmente se creó para liberalizar los sectores productivos, pero a la postre se convirtió en un decreto ley a propuesta del titular del Ministero dello Sviluppo Economico ${ }^{4}$, Pier Luigi Bersani, quien lo emitió el 4 de julio de 2006. El interés del Decreto Bersani era sentar las bases para que se legislase en torno a nuevas reglas que fomentasen un mercado más competitivo, tutelar los intereses de los consumidores y vigilar la competencia de los agentes. En segundo lugar, se ponía el acento en procedimientos más rigurosos contra los evasores fiscales. Este decreto se convirtió finalmente en la Ley 40 del 2 de abril de 2007. En los dos años que han transcurrido desde su publicación, su efecto positivo se ha hecho presente en acciones específicas en los sectores (véase el cuadro 3):

3. Autoridad Garante de la Competencia y del Mercado.

4. Ministerio del Desarrollo Económico. 


\begin{tabular}{|c|c|c|c|}
\hline 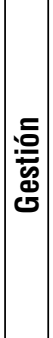 & 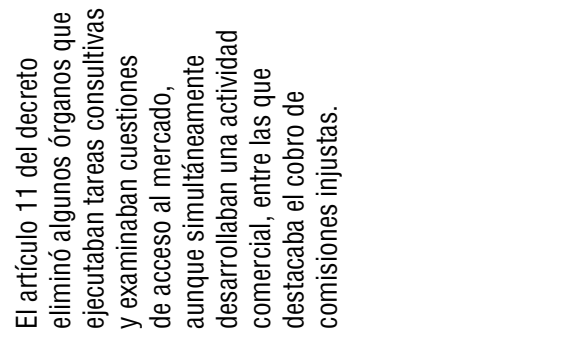 & 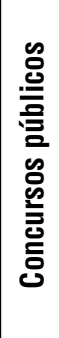 & 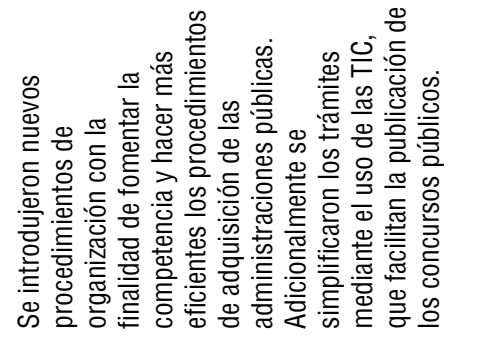 \\
\hline 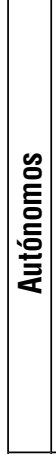 & 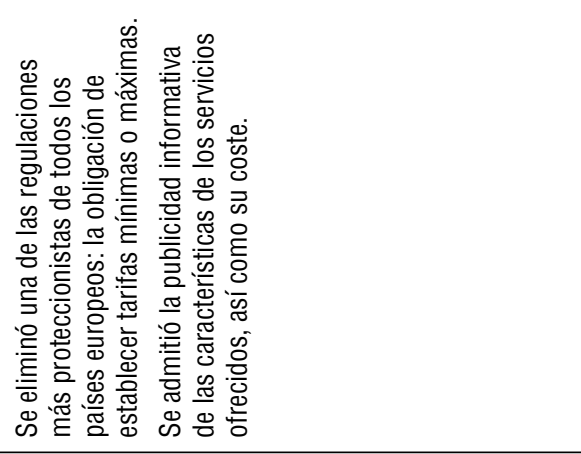 & 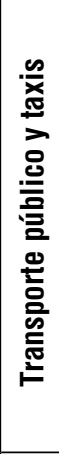 & 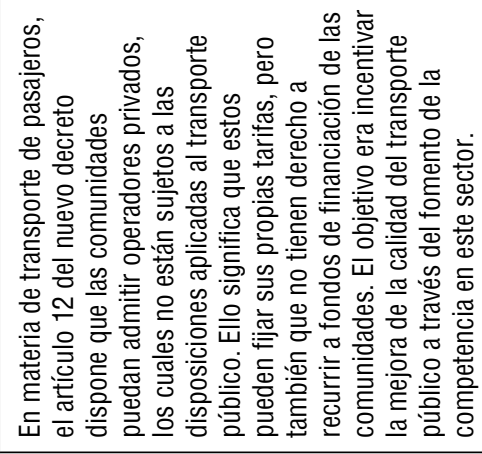 \\
\hline $\begin{array}{l}\text { 은 } \\
\text { 导 } \\
\text { 衣 }\end{array}$ & 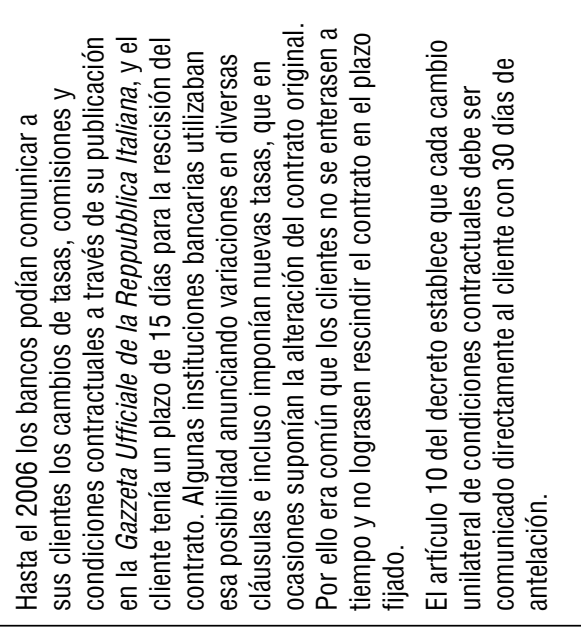 & 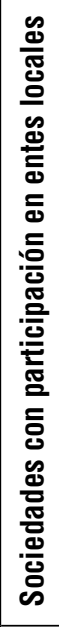 & 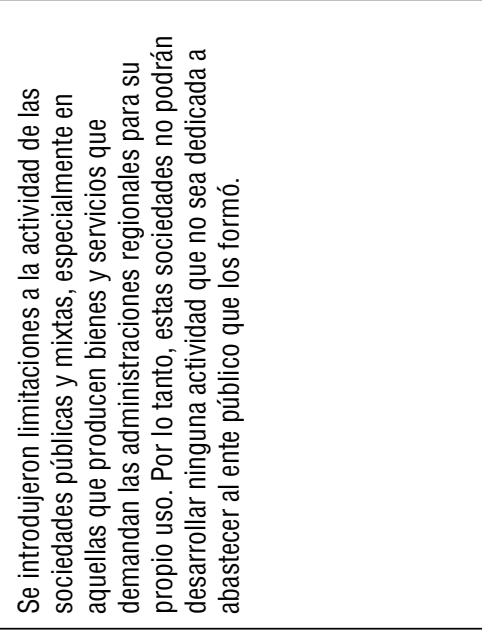 \\
\hline 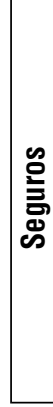 & 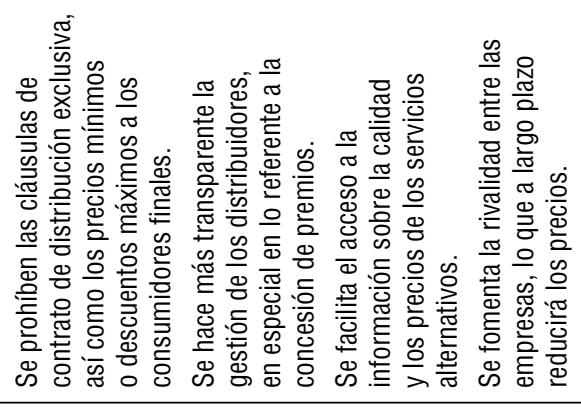 & 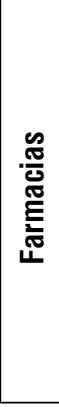 & 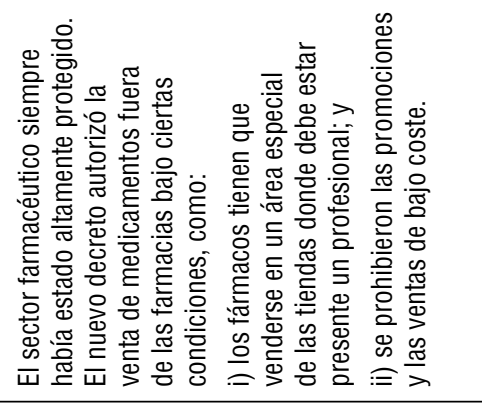 \\
\hline
\end{tabular}


i) de los seguros;

ii) de la banca;

iii) de los trabajadores autónomos;

iv) de la gestión (conocidos como comisiones de administración);

v) de la distribución de medicamentos (especialmente en las farmacias);

vi) de las sociedades con participación en entes locales;

vii) del transporte público y los taxis;

viii) de los concursos públicos, entre otros.

En materia de privatizaciones, en junio de 1993 el gobierno italiano se comprometió a emprender una serie de privatizaciones de empresas públicas sobre la base de tres propósitos específicos. El primero consistió en dar cabal cumplimiento a las condiciones de convergencia señaladas en el Tratado de Maastricht y, de ese modo, asegurarse un sitio en la Unión Económica y Monetaria (UEM). El segundo era reducir el déficit público (entonces superaba los 152 trillones de liras, equivalentes al $8 \%$ del PBI, en comparación con el 1,2\% del británico). Finalmente, el tercero era buscar que con las privatizaciones la actividad productiva italiana fuese más competitiva. Una muestra del elevado intervencionismo en la economía lo constituía el que por entonces el Estado era el propietario del $75 \%$ de la totalidad de las entidades bancarias. En la actualidad la cuota del mercado de las entidades estatales se ha reducido considerablemente, si bien las denominadas fundaciones públicas aún pertenecen a los institutos de crédito oficiales (véase OECD, 2008b). En el cuadro 4 se muestra una relación de las empresas privatizadas entre 1993 y 2002, que es justo el periodo de mayor actividad en la materia.
En julio de 2004, el MEF publicó un balance del proceso de privatización en el país. Los resultados fueron positivos porque la venta de las empresas estatales contribuyó a reducir el tamaño del sector público, y los ingresos captados por las arcas públicas han colocado a Italia en el segundo lugar entre los países occidentales en cuanto al valor de ingresos y en el primero a nivel europeo en la venta de empresas públicas (entre 1994 y 2003 el Estado ingresó un importe superior a los 90 mil millones de euros). Según Romano y Ferrari (2004), proporcionalmente en el 2003 Italia concentró el 34\% de las operaciones de privatización en todo el mundo, lo cual es muy significativo porque en 1997 solo concentraba el 14\% (en 1999 y en el 2001 fue del 15\%).

Dos de las privatizaciones más emblemáticas de los últimos tiempos han sido las del Ente Nazionale per L'energia Elettrica ${ }^{5}$ (ENEL) y del Ente Nazionale Idrocarburi ${ }^{6}$ (ENI). A finales de la década del noventa tuvo lugar el primer ejercicio de desregulación en el sector energético con la privatización parcial del monopolio estatal ENEL S.p.A; este proceso, sin embargo, estuvo lejos de ser modélico porque para mediados de la década actual el Estado todavía era el propietario del $68 \%$ de las acciones de la empresa (véase Ministero del Tesoro, Bilancio e Programmazione Economica, 2003). Las primeras presiones para que se privatizase ENEL tuvieron lugar en 1996, con el Decreto 79/99 emitido por el Consejo Europeo, en el que se señalaba la necesidad de que se separasen el patrimonio y la gestión de la red de transmisión eléctrica italianos y que se transfirieran a un ente público controlado por el MEF.

5. Ente Nacional para la Energía Eléctrica.

6. Ente Nacional de Hidrocarburos. 
Cuadro 4. Privatizaciones más emblemáticas en Italia entre 1993 y 2002

\begin{tabular}{|c|c|c|c|c|}
\hline & Empresa o grupo & Método de venta & $\begin{array}{c}\text { Porcentaje } \\
\text { vendido (en \%) }\end{array}$ & $\begin{array}{c}\text { Cantidad (en millones } \\
\text { de euros) }\end{array}$ \\
\hline \multirow[t]{5}{*}{1993} & Italgel & Acuerdo privado & 62,12 & 223 \\
\hline & Cirio-Bertolli_De Rica & Acuerdo privado & 62,12 & 160 \\
\hline & Crédito Italiano (IRI) & Oferta pública & 58,09 & 930 \\
\hline & SIV (EFIM) & Subasta & 100 & 108 \\
\hline & \multicolumn{3}{|c|}{ Total del año } & 1422 \\
\hline \multirow[t]{7}{*}{1994} & IMI (1. a parte) & Oferta pública & 32,89 & 927 \\
\hline & COMIT (IRI) & Oferta pública & 54,35 & 1493 \\
\hline & Nuevo Pignone (ENI) & Subasta & 69,33 & 361 \\
\hline & INA (1. ${ }^{\mathrm{a}}$ parte $)$ & Oferta pública & 47,25 & 2340 \\
\hline & Acciai Speciali Terni & Acuerdo privado & 100 & 322 \\
\hline & SME (1. ${ }^{\text {a }}$ parte $)$ & Acuerdo privado & 32,00 & 373 \\
\hline & \multicolumn{3}{|c|}{ Total del año } & 6377 \\
\hline \multirow[t]{9}{*}{1995} & Italtel & Subasta & 40 & 516 \\
\hline & Ilva Laminati Piani & Acuerdo privado & 100 & 1298 \\
\hline & Enichem Augusta (ENI) & Subasta & 70 & 155 \\
\hline & IMI (2. ${ }^{\mathrm{a}}$ parte $)$ & Acuerdo privado & 19,03 & 472 \\
\hline & SME (2. ${ }^{\text {a }}$ parte) & Aceptaron ofertas & 14,91 & 176 \\
\hline & INA (2. a parte) & Acuerdo privado & 18,37 & 871 \\
\hline & ENI (1. a parte) & Oferta pública & 15 & 3253 \\
\hline & ISE & Subasta & 73,96 & 191 \\
\hline & \multicolumn{3}{|c|}{ Total del año } & 7106 \\
\hline \multirow[t]{7}{*}{1996} & Dalmine & Subasta & 84,08 & 156 \\
\hline & Nuova Tirrena & Subasta & 91,14 & 283 \\
\hline & SME (3. ${ }^{\text {a }}$ parte) & Aceptaron ofertas & 15,21 & 62 \\
\hline & INA (3. a parte) & Oferta de los accionistas & 31,08 & 2169 \\
\hline & IMI (3. a parte) & Oferta pública & 6,94 & 259 \\
\hline & ENI (2. a parte) & Oferta pública & 15,82 & 4582 \\
\hline & \multicolumn{3}{|c|}{ Total del año } & 7742 \\
\hline \multirow[t]{6}{*}{1997} & ENI (3. ${ }^{a}$ parte) & Oferta pública & 17,60 & 6833 \\
\hline & Aeroporti di Roma & Oferta pública & 45 & 307 \\
\hline & Telecom Italia & Aceptaron ofertas & 39,54 & 11818 \\
\hline & SEAT Editoria & Oferta de los accionistas & 61,27 & 854 \\
\hline & Banca di Roma & Oferta pública & 36,50 & 980 \\
\hline & \multicolumn{3}{|c|}{ Total del año } & 20940 \\
\hline \multirow[t]{4}{*}{1998} & SAIPEM (ENI) & Oferta pública & 18,75 & 589 \\
\hline & ENI (4. a parte) & Oferta pública & 14,83 & 6711 \\
\hline & BNL & Oferta pública & 67,85 & 3464 \\
\hline & \multicolumn{3}{|c|}{ Total del año } & 10764 \\
\hline \multirow[t]{4}{*}{1999} & ENEL & Oferta pública & 31,70 & 16550 \\
\hline & Autostrade & Subasta y oferta pública & 82,40 & 6722 \\
\hline & Mediocrecito Centrale & Subasta & 100 & 2037 \\
\hline & \multicolumn{3}{|c|}{ Total del año } & 25382 \\
\hline
\end{tabular}


Cuadro 4 continuación

\begin{tabular}{|c|c|c|c|c|}
\hline $\mathbf{2 0 0 0}$ & Aeroporti di Roma & Venta directa & 51,2 & 1327 \\
\cline { 2 - 5 } & Finmeccanica & $\begin{array}{c}\text { Oferta pública } \\
\text { secundaria }\end{array}$ & 43,7 & 5505 \\
\cline { 2 - 5 } & COFIRI & Venta directa & 100 & 504 \\
\cline { 2 - 5 } & Banco di Napoli & $\begin{array}{c}\text { Oferta directa a los } \\
\text { accionistas }\end{array}$ & 16,2 & 493 \\
\cline { 2 - 5 } & \multicolumn{3}{|c|}{ Total del año } & $\mathbf{7 9 3 3}$ \\
\hline $\mathbf{2 0 0 1}$ & ENI (5. ${ }^{\text {parte) }}$ & Venta rápida en bloque & 5 & $\mathbf{2 9 0 7}$ \\
\hline \multirow{2}{*}{2002} & Total del año & 1400 \\
\hline
\end{tabular}

Fuente: Ministerio del Tesoro, Bilancio e Programmazione Económica (2003).

ENEL fue obligada a vender la mayoría de sus derechos sobre las redes eléctricas; al poco tiempo afloraron grandes problemas de coordinación entre el gerente de la red y su propietario, debido a lo cual a partir de 2005 el gobierno decidió reunificar la propiedad y la gestión. El siguiente paso fue crear Trasmissione Elettricità Rete Nazionale S.p.A $\mathrm{A}^{7}$ (Terna), a la cual se le cedió la mayor parte de la red de transmisión. Hoy en día Terna es la propietaria de más del $90 \%$ de las redes de transmisión (el restante $10 \%$ está distribuido entre trece empresas municipales, todas de mediano tamaño). En la actualidad Terna forma parte de la empresa estatal Cassa Depositi e Prestiti ${ }^{8}$ (CDP), propietaria de las acciones de ENEL (a su vez, esta posee acciones de Terna). Como se puede ver, el mercado eléctrico italiano está lejos de operar de manera competitiva porque una inmensa mayoría de las empresas dependen de que ENEL les permita el acceso a sus redes (véase Autorità per l'Energia Elettrica e il Gas, 2006).

Por su parte, ENI S.p.A es la petrolera italiana más grande, con unas ventas anua-

7. Red Nacional de Transmisión de Electricidad.

8. Caja de Depósitos y Préstamos. les de 86100 millones de euros y con unos beneficios netos de 9200 millones de euros en el 2007. Esta empresa es la octava a nivel europeo, da empleo a más de 70 mil trabajadores repartidos en 70 países y ostenta el $29,3 \%$ de la cuota de mercado, lo que la hace líder en su país de origen. Con su propia red de estaciones de servicio opera con la marca Agenzia Generale Italiana di Petroli $^{9}$ (AGIP), fundada en 1953, la cual posee la red más amplia de estaciones de servicios y del suministro de gas en el país. En 1992 se convirtió en sociedad anónima y, finalmente, entre 1995 y 1998 se emitió al mercado el $62 \%$ de sus acciones. En el gráfico 1 se aprecia la forma como están repartidas las acciones de esta empresa. En la actualidad el Estado italiano aún posee el $30 \%$ de las acciones (lo que lo constituye todavía como el accionista mayoritario), el $8 \%$ está en manos de sus trabajadores y el restante $62 \%$ en el mercado.

Para la OCDE (2007), el Estado, consciente de la necesidad de eliminar el monopolio del que gozaba ENI, promovió la sentencia legislativa 625/96, en la que

9. Agencia General Italiana de Petróleos. 
Gráfico 1. Reparto de las acciones de ENI

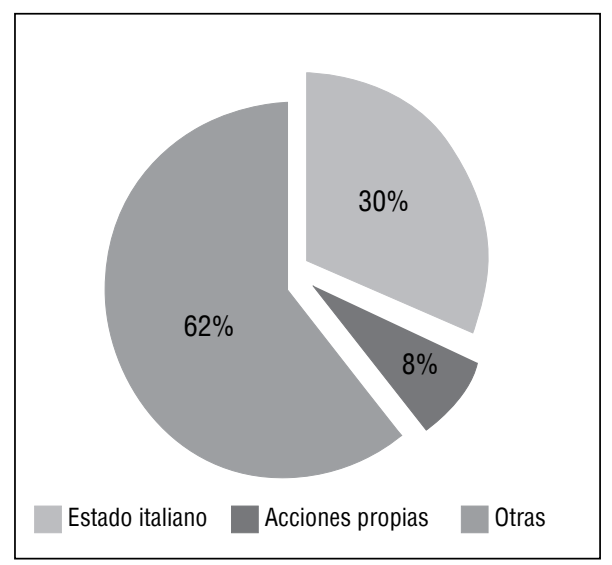

Fuente: Portal web de ENI.

se señala que este quedaba abolido. Si bien esto ha sido un notable avance, ciertamente aún prevalece un grave conflicto de intereses porque, por una parte, el Estado en su calidad de accionista está interesado en recibir dividendos y maximizar sus beneficios, pero, por otra parte, está obligado a asegurar el abastecimiento de gas en los hogares y combustible en las estaciones de servicio. En torno a esta cuestión los ciudadanos italianos acusan al Estado de utilizar a ENI como una fuente oculta de recaudación tributaria.

La privatización de ENEL y ENI ha sido muy significativa, pero ciertamente todavía hay un largo camino por recorrer. En la actualidad el Estado aún es el accionista mayoritario de varias grandes empresas, como Finmeccanica S.p.A (con el 32,3\%), Poste Italiane (con el 65\%), o Anas S.p.A, Ferrovie dello Stato y RAI Holding (todas ellas con el 100\%), entre otras (véase Romano y Ferrari, 2004). Según la OCDE (2007), la influencia y el control que ejerce el gobierno italiano es notablemente significativo en las operaciones comerciales y sobre la gestión de las propias empresas.

\section{Lucha contra los monopolios}

Con la entrada en vigor del Tratado de Roma (en 1958), los Estados miembros acordaron establecer unas normas comunes en materia de defensa de la competencia, tal y como queda explícitamente señalado en sus artículos 81 y 82 , que prohíben las acciones que atenten contra la competencia y los abusos de posiciones dominantes. Desde entonces se han desarrollado diversas leyes para garantizar la competencia entre los países miembros. Del cúmulo de leyes legisladas recientemente en este ámbito, destacan las regulaciones emitidas por el Council Regulations ${ }^{10}$, al amparo de la Comisión Europea. Tal es el caso del Decreto $1 / 2003^{11}$, que reconoce la aplicación de las leyes que regulan la justa $y$ transparente competencia entre los países miembros; asimismo, con este decreto se potenció la colaboración entre las instituciones nacionales competentes. En lo referente al abuso de posición dominante, el decreto en cuestión reconoce que se produce cuando una empresa alcanza un poder económico tal, que obstaculiza la competencia eficiente en el mercado, y señala que las empresas que ostentan una posición dominante están obligadas a permitir el desarrollo del mercado y la entrada de nuevos competidores.

El 10 de octubre de 1990, mediante la Ley 287, se creó la AGCM, a la que se le dotó de plena independencia. La AGCM se forma de un órgano colegiado, el cual está integrado por un presidente y cuatro miembros, elegidos por los presidentes del Senado y del Congreso. Esta institución es la responsable de vigilar el buen funcionamiento de los agentes económicos

10. Consejo de las Regulaciones.

11. En vigor desde enero de 2004. 
que operan en el mercado, y de ese modo garantizar la libre competencia, detectar abusos de posiciones dominantes, supervisar prácticas comerciales y publicitarias $\mathrm{y}$, muy especialmente, detectar conflictos de intereses.

Unos años más tarde, en la Ley 249 de 1997 adicionalmente se crearon dos organizaciones similares, pero especialmente dirigidas a vigilar las telecomunicaciones y el sector de la energía:

i) la Autorità per le Garanzie nelle Comunicazioni $^{12}$ (AGCom); y

ii) la Autorità per l'Energia Elettrica e il $\mathrm{Gas}^{13}$ (AEEG).

Recientemente a través de la Ley 223 de 2006 se han ampliado los poderes de la AGCM con la introducción de nuevos instrumentos que desde hace tiempo existen a nivel comunitario, como:

i) extender su margen de acción;

ii) se la ha dotado de nuevas medidas de prevención en caso de producirse un riesgo de daño grave o irreversible para la competencia o el mercado; y

iii) un programa de indulgencia para aquellos agentes que denuncien prácticas monopolísticas y de ese modo puedan ver reducidas las sanciones.

A la luz de los resultados logrados al día de hoy, se puede decir que el trabajo realizado por la AGCM ha sido positivo, si bien queda mucho por hacer. Es necesario redoblar esfuerzos especialmente en los casos de las empresas que en el pasado eran monopolios u oligopolios estatales, que

12. Autoridad para la Garantía en las Comunicaciones.

13. Autoridad para la Energía Eléctrica y el Gas. se transformaron en sociedades anónimas o que fueron privatizadas, pero que aún concentran un elevado poder de mercado. Según los informes publicados por la propia AGCM, en los últimos siete años se han multiplicado los casos registrados. A modo de ejemplo, entre el 2006 y 2007 se verificó un aumento del $20 \%$ de empresas que cometieron alguna violación de las normas de la competencia. Por el lado contrario, hubo una notable reducción del número de concentraciones prohibidas o aceptadas de forma condicionada.

El cuadro 5 muestra una selección del número de empresas investigadas por la AGCM desde su creación. Los datos más significativos se centran en torno al número de empresas que fueron objeto de investigación. En el ámbito de las prácticas monopolísticas se concentró el mayor número de ellas (864 en el 2007), mientras que en menor número se encontraron las que violaron alguna ley específica (solo dos en ese mismo año). En términos generales, dos de cada cinco casos entran en el apartado correspondiente a las violaciones de leyes.

Dos de los casos más relevantes que analizaron las autoridades de la competencia italianas son los de Mediaset y Telecom Italia S.p.A. El caso de Mediaset fue especialmente complejo por varias razones. Es de dominio público que el actual primer ministro italiano, Silvio Berlusconi, es el poderoso líder de la formación política Il Popolo della Libertà ${ }^{14}$ (que es la sucesora de la antigua Forza Italia), pero también es la cabeza indiscutible del emporio empresarial Fininvest, que engloba algunos de los medios de comunicación más importantes del país (como el poderoso grupo Mediaset,

14. El Pueblo de la Libertad. 


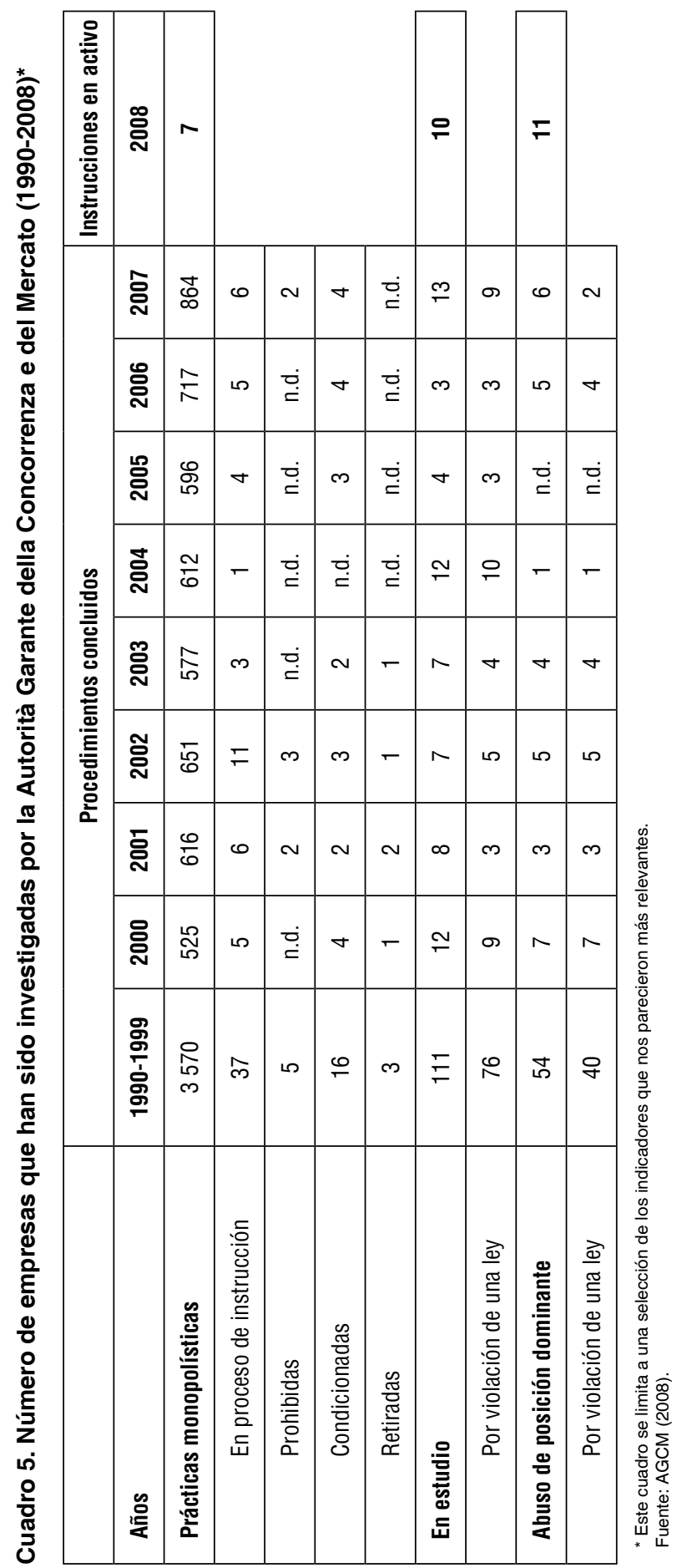


en $36 \%$, o de Mondadori, que posee editoriales, periódicos y empresas de radio), así como clubes de fútbol (el 100\% del A.C. Milan), empresas de entretenimiento (el $100 \%$ del teatro Manzoni), empresas de servicios financieros (el 35\% de Mediolanum), entre otras.

Por esta razón, en múltiples ocasiones Berlusconi ha sido acusado de interferir en la acción de la justicia por actuar en su favor aun en contra de los intereses del Estado y por corrupción. Por estas y otras razones ha sido acusado ante los tribunales, en algún caso incluso ha sido condenado, pero nunca ha sido encarcelado (véase CIDOB, 2008). Por ello resulta especialmente relevante que en Italia los medios de comunicación sean independientes y que reflejen la variedad de opiniones de los distintos partidos políticos.

Para Padovani (2008), en Italia la televisión desempeña un papel, si cabe, más importante que en el resto de países comunitarios porque la mayoría de los ciudadanos italianos se informa exclusivamente a través de los telegiornale (telediarios). Una prueba que lo constata es que los periódicos italianos tienen una tirada que en ningún caso supera los 5 millones, en comparación con los 28 millones en Alemania. Desde hace dos décadas el mercado televisivo se caracteriza por ser un oligopolio: solo hay canales estatales de la $\mathrm{RAI}^{15}$ y los canales privados de Mediaset.

En el ocaso del primer gobierno de Berlusconi, la coalición de partidos de centroizquierda L'Ulivo (El Olivo) denunció que el conjunto de conflictos de interés situó a Italia en el lugar 77 en el ránking que

15. Radiotelevisione Italiana, antiguamente conocida como Radio Audizioni Italiane. representa la libertad de prensa mundial, elaborado por el Freedom House (2007), y fue acreedora a la calificación «Partly Free» (parcialmente libre). Según su informe Freedom of the Press que publica este organismo entre 1980 y 1993, la libertad de prensa en Italia obtuvo una calificación aprobatoria (en todos los años se le dio la nota de «Free»). En 1994, sin embargo, el país ocupó el puesto 25 en el ránking de libertad de prensa, un año después bajó al puesto 30, y entre 1999 y el 2003 osciló entre el puesto 27 y 28, pero en el 2004 bajó al puesto 33, y, finalmente, la peor nota la obtuvo en el bienio 2005-2006, con el puesto 35 (España ocupó el puesto 21). Resulta interesante constatar que desde que Silvio Berlusconi se ha consolidado en el poder como primer ministro de su país, la libertad de prensa cada vez está peor valorada. Como lo señala Della Porta (1996), los adversarios de Berlusconi lo acusan, entre otros delitos, de haber pagado en 1997 al abogado británico David Mills la cantidad de 600 mil dólares en recompensa por no haber testificado en su contra en dos procesos sobre dos sociedades presuntamente ilegales, ambas propiedades de Mediaset.

En el 2001 Rossend Domènech Matilló, en un trabajo realizado para la Fundación Campalans, expresaba su preocupación de que el entonces primer ministro Berlusconi promoviese la legislación de leyes que fuesen favorables a sus intereses particulares y los de sus empresas. Unos años más tarde, la Università Carlo Cattaneo (2008) publicó un estudio en el que señalaba que a mayor cantidad de horas frente al televisor, era más probable que los electores votasen por Berlusconi. A la luz de los resultados arrojados en las elecciones de abril de 2008, la coalición encabezada por Berlusconi, el Popolo della Libertà, ganó al representante de la coalición de partidos, el socialista 
Walter Veltroni, tal y como lo vaticinaba el estudio de la Università Carlo Cattaneo. Cabe la duda de saber si en los días previos a las elecciones los italianos vieron demasiada televisión.

El segundo caso que nos llama la atención es el de Telecom Italia S.p.A. Esta empresa fue creada en 1990 tras la fusión de todas las empresas públicas del sector de las comunicaciones, incluidos tanto proveedores de servicios como de manufacturación. En 1995 se adhirió el primer operador móvil Telecom Italia Mobile. Dos años después, en 1997, el gobierno creó la AGCom, a la cual se le dotó de plena independencia, se le designó la responsabilidad de vigilar el proceso de privatización de Telecom Italia S.p.A, de tutelar la liberalización del sector, de garantizar el fomento de nuevas telecomunicaciones y de potenciar el acceso a las redes de comunicación. Por ese entonces el segmento de telefonía fija estaba dominado por una sola empresa, debido, entre otras cuestiones, a la escasez de licencias para que participasen otros operadores, mientras que en el segmento de telefonía móvil operaban tres empresas distintas.

Pero la posición dominante de Telecom Italia S.p.A apenas si ha variado desde entonces, en particular en los mercados de la telefonía de voz y el de las conexiones de banda ancha. Según la AGCM (2006), esta empresa sigue siendo la líder indiscutible gracias a siete aspectos relevantes:

i) es la propietaria de la más amplia red de telefonía a nivel nacional y, por lo tanto, sus competidores dependen de su política de precios para acceder a ella;

ii) goza de una gran aceptación entre los usuarios de sus productos y servicios, generalmente reacios a cambiarse de la compañía que les ha dado el servicio durante muchos años; iii) tiene mayor capacidad para generar innovaciones, por lo cual puede ofrecer una amplia gama de productos y servicios;

iv) aprovecha su capacidad para generar economías de escala;

v) se ha resistido a eliminar barreras de entrada;

vi) cuenta con un valioso patrimonio, que es la información privilegiada que se deriva de la posición monopolista que detentó en el pasado, lo que implica una notable ventaja competitiva en relación con sus competidores: es la propietaria de la base de datos histórica sobre el tráfico, la intensidad de uso de los servicios telefónicos y sobre el comportamiento de los consumidores, lo que la faculta para diseñar mejores estrategias de publicidad; $y$

vii) en el caso del mercado de ADSL ${ }^{16}$, hasta el 2006 concentró el 85\% del mercado a nivel nacional y el $70 \%$ en zonas con mayor cantidad de oferentes alternativos; sin embargo, el restante $30 \%$ también eran consumidores indirectos de Telecom, porque mayoritariamente utilizaban accesos ULL (Unbundling Local Loop), que eran proporcionados por esta empresa al oferente alternativo (lo cierto es que algunas de estas prácticas pueden ser consideradas de abuso de posición dominante y de intento de exclusión de la competencia). Como es natural, el resultado de todo lo anterior es que a efectos prácticos Telecom goza de una posición imbatible en los mercados minorista y mayorista.

16. Asymmetric Digital Subscriber Line (Línea de Suscripción Digital Asimétrica, más conocida como "banda ancha"). 
Cuadro 6. Participación en los mercados de telefonía y banda ancha

\begin{tabular}{|c|c|c|}
\hline Cuota de mercado (en \%) & Telefonía & Banda ancha \\
\hline Telecom Italia & 62,5 & 57,5 \\
\hline Wind & 10,4 & 8,0 \\
\hline Fastweb & 2,4 & 20,5 \\
\hline BT Italia & 8,3 & 3,3 \\
\hline Tele 2 & 7,8 & 2,0 \\
\hline Tiscali & 1,6 & 0,4 \\
\hline Altri & 7,0 & 8,3 \\
\hline
\end{tabular}

Fuente: AGCom (2008).

Telecom es la empresa líder del sector, de lo que da fe la propia AGCom (2008) en el cuadro 6; sin embargo, en principio en el 2008 empezó a haber una cierta tendencia al equilibrio. Como lo advertimos anteriormente, en el 2006 esta empresa concentraba el $85 \%$ del mercado, pero dos años más tarde se redujo al 57,5\%. Su competidor más cercano, Fastweb, solo ostenta el 20,5\% y aun más atrás están Altri y Wind, con el $8,3 \%$ y el $8 \%$, respectivamente (el resto es insignificante). Dicho esto, no cabe duda del arrollador poder de Telecom, pero quisiéramos permitirnos pensar que las cosas van por buen camino.

\section{Lucha contra la piratería y el contrabando}

Italia ostenta las tasas de piratería más elevadas de los países miembros de la UE-15. En los últimos años han crecido de manera extraordinaria la piratería en Internet y la venta de productos piratas a pie de acera. Para Bagnasco Petrelli ${ }^{17}$ (2008), los responsables de este incremento han sido las

17. Profesor de la Universidad de Padua. autoridades (funcionarios, legisladores y jueces), los consumidores (en este país la compra de productos piratas y en general de contrabando no está socialmente mal visto) y los sindicatos del crimen organizado, especializados en prácticamente toda la cadena del negocio, que va desde la grabación ilegal de películas, documentales, conciertos, representaciones teatrales hasta partidos de fútbol, así como la edición, reproducción en formato digital y la distribución a través de redes (que usualmente también se utilizan para distribuir drogas y armas), hasta el "alquiler" de los espacios en plena calle para que los "sin papeles" vendan los CD y DVD, así como las imitaciones de los bolsos de Gucci, Louis Vuitton y Prada, la ropa y los perfumes de Armani, Versace y Valentino, o la peletería de Gianfranco Ferré y Salvatore Ferragamo.

La piratería en Italia ha llegado a tal extremo, que recientemente también ha incursionado en el ámbito de la edición de libros (posiblemente los libros que más han sufrido el acoso de la piratería han sido la novela El código Da Vinci, del escritor Dan Brown, y las diversas entregas de 
Harry Potter, de la escritora británica J.K. Rowling) y revistas (una de las principales víctimas ha sido The Rolling Stones), la adulteración de bebidas alcohólicas (en las calles de Nápoles o Roma fácilmente se pueden adquirir botellas de coñac, whisky y hasta tequila, cuando en realidad su contenido es alcohol adulterado, pero que ha sido envasado en botellas originales recuperadas de los vertederos de basura). El colmo de la desfachatez es que también han falsificado y circula por los circuitos del comercio ilegal la novela Gomorra, del autor Roberto Saviano. Este libro ha vendido 1,5 millones de copias solo en Italia ( 250 mil de ellas fueron piratas), ha sido traducido a 33 lenguas y ha llegado a los escaparates en 42 países. Gomorra de manera novelada cuenta cómo funciona la camorra (véase The Heritage Foundation and Wall Street Journal, 2008).

Según el Index of Economic Freedom ${ }^{18}$ de 2008, Italia ocupa el puesto 29 en el indicador de corrupción entre los 41 países que fueron objeto de análisis. Este dato sirve de argumento a la Heritage Foundation para afirmar que esta posición se explica porque algunos de los más altos cargos del gobierno están en manos de funcionarios corruptos, quienes fomentan la piratería en lugar de luchar contra ella.

El cuadro 7 muestra el país de origen de los productos piratas y del contrabando en el 2004. En todos los segmentos analizados, China ocupa el primer lugar. Llaman particularmente la atención los casos de perfumería y cosméticos (con el $100 \%$ ), juguetes y juegos (92\%) y los CD y DVD (87\%). Otros países relevantes son Túnez, en alimentos y bebidas (con el $100 \%$ ), y Tailandia y Hong Kong porque

18. Índice de Libertad Económica. están presentes en casi todos los segmentos. Los resultados totales de 2004 señalan que China fue el origen del $72 \%$ de los productos piratas y del contrabando, Tailandia del $8 \%$ y Hong Kong del 5\%.

Lo peor de esta situación -así lo denunció un informe que publicó la International Intellectual Property Alliance ${ }^{19}$ (IIPA, 2007) - es que el gobierno italiano ha promovido algunas reformas sobre las leyes de copyright, que lejos de proteger perjudicaban los derechos de autor. Las leyes a las que se refiere este informe son:

i) Ley de Indulto (de 2006), en la cual se concedió amnistía a delincuentes que cometieron delitos contra los derechos de autor cuyas penas carcelarias no superaban los tres años;

ii) Ley Cirelli (de 2005), sobre la base de la cual se archivaron cientos de casos antiguos que no habían sido resueltos; $\mathrm{y}$

iii) Ley Pecorella (de 2006), en la cual se eliminaron algunos derechos de la parte agraviada para impugnar sentencias judiciales o de proyectos legislativos que les perjudicasen.

Sin embargo, debido a la alarma social que generaron estas disposiciones legales, previsiblemente sean reformadas en los siguientes criterios:

- Hacer más expedita la tramitación de leyes que tutelen mejor todo lo referente al uso de las tecnologías de la información y la comunicación (TIC).

- Legislar en torno a las redes de inter-

19. Alianza Internacional de Propiedad Intelectual. 


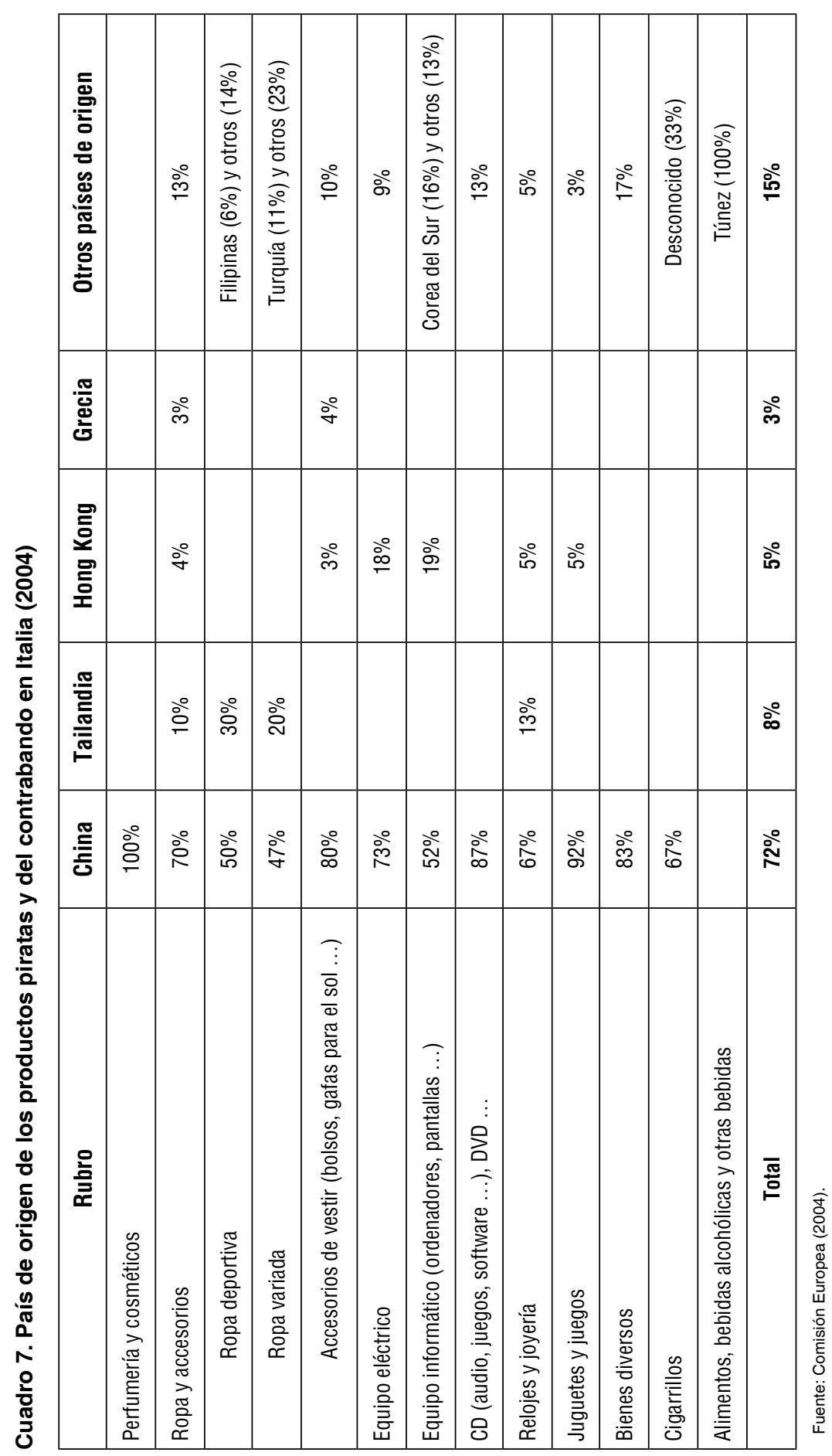


cambio de archivos (P2P) y combatir las actividades que atenten contra los derechos de autor.

- Emprender una campaña a nivel nacional contra la piratería y hacer especial hincapié en que esta es controlada por organizaciones criminales.

- Combatir el robo de imágenes y sonido en eventos culturales y deportivos.

- Generar estrechos lazos de cooperación entre la Direction Nationale Antimafia $^{20}$ (DNA) y Direzione Investigativa Antimafia ${ }^{21}$ (DIA), para el intercambio de información en materia de piratería y contrabando, con especial énfasis en las actividades delictivas promovidas por la $\mathrm{Ca}$ morra.

- Se alentará para que las empresas proveedoras de los servicios de ADSL creen un código deontológico que regule las relaciones entre ellas.

- Hacer una campaña a nivel nacional para que los ciudadanos asuman una mayor conciencia sobre la importancia del respeto a los derechos de autor, muy específicamente en el entorno on line.

Cuadro 8. Ránking de países por el volumen de negocio en el sector musical en 1996

\begin{tabular}{|c|c|c|c|}
\hline Principales países & $\begin{array}{c}\text { Ventas legítimas } \\
\text { (en millones de dólares) }\end{array}$ & $\begin{array}{c}\text { Ventas piratas } \\
\text { (en millones de dólares) }\end{array}$ & $\begin{array}{l}\text { Ventas piratas como } \\
\text { porcentaje del total de } \\
\text { unidades vendidas }\end{array}$ \\
\hline Rusia & 230 & 350 & 70 \\
\hline Brasil & 1394,5 & 200 & 45 \\
\hline China & 177,5 & 165 & 54 \\
\hline Italia & 637,5 & 105 & 22 \\
\hline India & 298 & 100 & 30 \\
\hline México & 399,3 & 70 & 50 \\
\hline Argentina & 285,3 & 65 & 30 \\
\hline Arabia Saudí & 100,9 & 35 & 30 \\
\hline Grecia & 128,7 & 22 & 25 \\
\hline Malasia & 99,9 & 18 & 20 \\
\hline \multicolumn{4}{|c|}{ Total mundial } \\
\hline Ventas & 39800 & 5000 & $12,5 \%$ \\
\hline Unidades & 4000 & 1500 & $33 \%$ \\
\hline
\end{tabular}

Fuente: OECD (2008a).

20. Dirección Nacional Antimafia.

21. Dirección Investigativa Antimafia. 
Ciertamente todos los esfuerzos que se emprendan a favor de hacer valer los derechos de propiedad intelectual son importantes. Como lo señala la OECD, desde mediados de la década del noventa, Italia se ha distinguido por ocupar los primeros puestos en materia de piratería de música, solo detrás de Rusia, Brasil y China.

Una década después estos datos no han mejorado en absoluto. En el 2007 más de 7 millones de italianos utilizaron redes de intercambio de archivos (P2P) para bajarse música en lugar de pagar por ella (IIPA, 2008). En términos generales, en todo el país la gente está poco sensibilizada con la piratería de archivos de música, pero el problema es más agudo en las ciudades del sur, de entre las cuales destaca Nápoles, donde no solo se trafican intensamente los productos piratas, sino que además se concentra el mayor número de grandes centros ilegales de grabación y distribución de copias ilegales.

Según la OCDE (2008a), la sede de la mayoría de estas redes se encuentra en manos de la Camorra, y en menor medida de la 'Ndrangheta calabresa, de la Cosa Nostra siciliana y de la Sacra Corona Unita. Solo la Camorra se estima que factura una cifra que se acerca a los 30 mil millones de euros al año, y la suma de todas estas organizaciones algunos años ha superado los 100 mil millones de euros; en realidad sus negocios abarcan un amplio abanico de actividades, como tráfico de drogas, extorsión y usura, tráfico de armas, tráfico de personas y prostitución $\mathrm{y}$, desde luego, piratería y contrabando.

En el 2007 estas organizaciones delictivas - sumadas las que están especializadas en la piratería y el contrabando- generaron a la industria nacional unas pérdidas que superaron los 1770 millones de dólares estadounidenses. En el cuadro 9 se puede apreciar la dimensión del negocio. Desde el 2003 el sector de software de negocios ha sido el más perjudicado; en el 2005, por ejemplo, 53\% del software especializado vendido en todo el país era falsificado. No muy lejos está el software de entretenimiento, que en el 2007 alcanzó una cifra estimada de 817 millones de dólares estadounidenses, con el $64 \%$ de las copias cuyo origen era ilícito. Finalmente, en el 2007 Italia ocupó el tercer puesto de piratería on line (Francia ocupó el cuarto y España el quinto) (IIPA, 2008).

Entre los diversos productos que son objeto de la piratería, llama especialmente la atención el caso del contrabando de cigarrillos. La Guardia di Finanza (GDF, equivalente a la policía fiscal, dependiente del MEF) es la responsable de combatir los delitos económicos. La GDF tiene un despliegue presencial en tierra, mar y aire; dado que Italia es una península las asociaciones delictivas suelen introducir la mercancía vía marítima, razón por la cual tiene bases en todo el territorio nacional, que abarca desde los Alpes, en la frontera con Suiza, hasta Pozano, en Sicilia, que es el puerto más cercano a Malta. El contrabando de tabaco está considerado uno de los más activos y lucrativos, en parte porque los impuestos a los cigarrillos en Italia son muy elevados.

La Commissione Parlamentare d'Inchiesta sul Fenomeno della Mafia e delle altre Associazioni Criminali Similari ${ }^{22}$ publicó un informe el 16 de julio de

22. Comisión parlamentaria de investigación contra la mafia. 


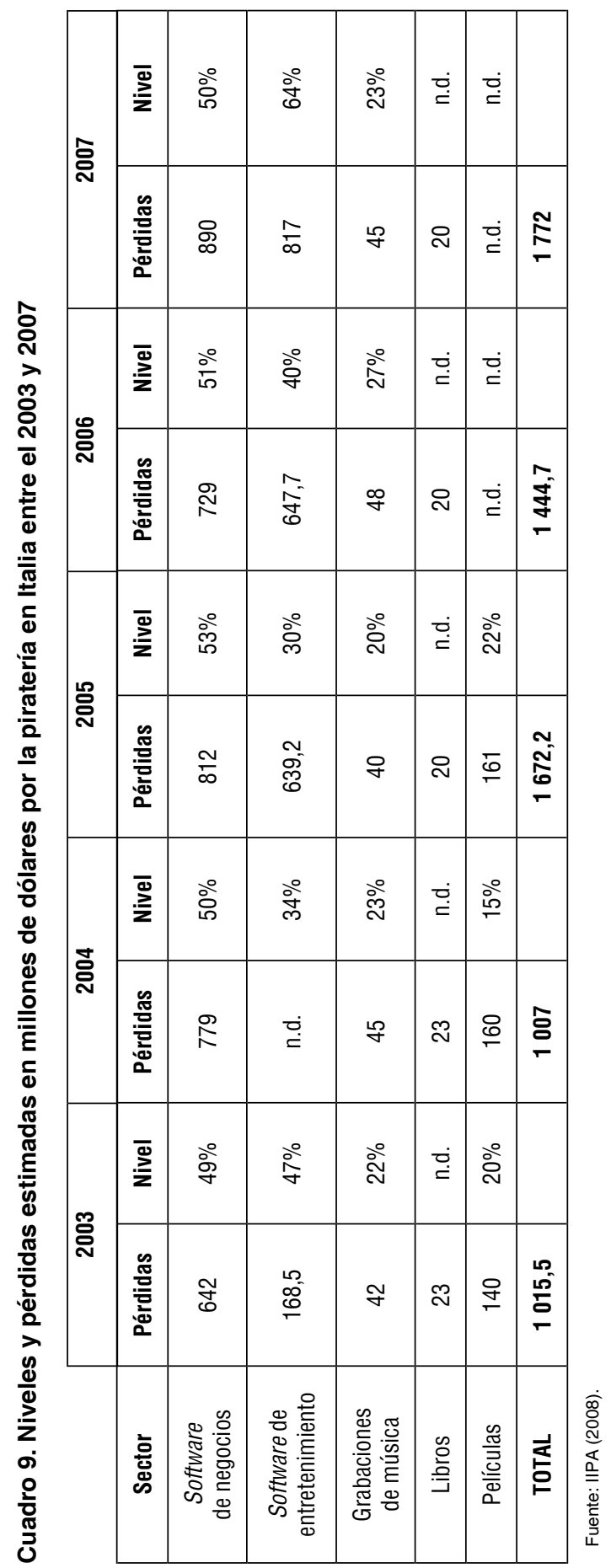


1999, en que señalaba que el contrabando de cigarrillos de marcas internacionales era la principal fuente de negocios de las organizaciones criminales de la época. Las investigaciones realizadas por esta comisión indicaban que productores internacionales de la talla de Philip Morris y Reynolds Tobacco Company, desde mediados de la década del ochenta, dirigían operaciones de importación a través de tres empresas suizas (Balmex AG, Basilio AG y Algrado $\mathrm{AG}$, todas ellas con oficinas en la ciudad de Basilea), las cuales mantenían estrechas relaciones con el crimen organizado italiano.

Ellos operaban fabricando cigarrillos con licencia en Suiza, los transportaban a Albania y posteriormente los introducían en Italia en lanchas rápidas. De ser cierta esta información, sería francamente lamentable que uno de los productores de tabaco más grandes y exitosos del mundo, como Philip Morris, no hubiese tenido escrúpulos en realizar negocios con las organizaciones criminales italianas. La cuestión es que, en todo caso, estos negocios fueron posibles en parte debido a la falta de estabilidad política que ha imperado en el país desde entonces.

\section{Conclusiones}

La economía italiana es considerada "libre" en $62,5 \%$, lo que la sitúa en la posición 64 entre las economías más libres del mundo. Alcanza buenos resultados en materia de libertad de negocio, comercio e inversiones y libertades laborales, pero el Estado aún es inmenso, lo que se ha traducido en una gestión torpe y cara del aparato de gobierno, pese a que en materia de privatizaciones Italia ocupa el segundo puesto en cuanto al valor de ingresos y en el primero a nivel europeo en la venta de empresas públicas.

Asimismo, Italia ostenta las tasas de piratería más elevadas de los países miembros de la UE-15 y ocupa el puesto 29 en el indicador de corrupción entre los 41 países que fueron objeto de análisis. Lo peor de esta situación es que el gobierno italiano ha promovido algunas reformas sobre las leyes de copyright, que lejos de proteger perjudican los derechos de autor. Las actividades derivadas de la piratería y del contrabando generaron a la industria nacional, en el 2007, unas pérdidas que superaron los 1770 millones de dólares estadounidenses. 


\section{Referencias bibliográficas}

AUTORITÀ GARANTE DELLA CONCORRENZA E DEL MERCATO (AGCM). 1990. Legge $N$. $^{\circ} 287$. Roma.

—. 2006. Informe Annuale 2006. Roma.

_. 2008. Annual Report. Roma.

AUTORITÀ PER LE GARANZIE NELLE COMUNICAZIONI (AGCom). 2008. Relazione Annuale, Roma.

\section{AUTORITÀ PER L'ENERGIA ELETTRICA E} IL GAS. 2006. Relazione annuale alla CE. $<$ http://www.autorita.energia.it/pubblica zioni/index.htm04/08/2006>.

BAGNASCO PETRELLI, Fabio. Entrevista telefónica realizada el 27 de octubre de 2008. Catriacalà, A. 2006. Relazione sull'attività svolta nel 2006, Liberalizzazione, semplificazione amministrativa e tutela dei consumatori. Milano: Roncagliolo.

CENTRO DE INVESTIGACIÓN DE RELACIONES INTERNACIONALES Y DESARROLLO (CIDOB). 2008. Silvio Berlusco$n i$. Barcelona: Fundación CIDOB. <http:// www.cidob.org/en/the_foundation>.

COMISIÓN EUROPEA (European Comission). 2004. Counterfeit Piracy Statistics. Bruxelles: EC.

2008. Tratados Constitutivos. Bruselas.

COMMISSIONE PARLAMENTARE D'INCHIESTA SUL FENOMENO DELLA MAFIA E DELLE ALTRE ASSOCIAZIONI CRIMINALI SIMILARI. $<$ http://www.parlamento.it/parlam/bicam/mafia/home.htm>.

COUNCIL REGULATIONS. 2004. Official Journal of the European Union. Jan. 1.
DELLA PORTA, Donatella. 1996. Partidos políticos y corrupción. Reflexiones sobre el caso italiano. Nueva Sociedad, N. ${ }^{\circ} 145$, sep.-oct. Págs. 92-109.

DOMÈNECH MATILLÓ, R. 2001. Elecciones italianas. Italia, laboratorio de la modernidad politica. Barcelona: Fundación Rafael Campalans.

ECONOMIC FREEDOM OF THE WORLD. 2007. Annual Report. <http://www.freethe world.com/2007/3EFW2007ch3.pdf>.

ENTE NAZIONALE IDROCARBURI S.p.A (ENI). <http://www.eni.it/en_IT/company/ company_swf.page $>$.

EUROPEAN INDUSTRIAL RELATIONS OBSERVATORY. 1999. Privatisation and Industrial Relations in Italy. <http://www. eurofound.europa.eu/eiro/1999/12/word/ it9909254s.doc>.

FREEDOM HOUSE. 2007. Freedom of the Press: Historical Data. $<$ http://www.freedom house.org/template.cfm?page $=274>$.

GOLDSTEIN, Andre. 2003. Privatization in Italy 1993-2002: Goals, Institutions, Outcomes, and outstanding Issues. CESifo Working Paper n. ${ }^{\circ} 912$.

HERITAGE FOUNDATION. 2008. Index 2008. $<$ http://www.heritage.org/research/features/ index/country.cfm?id=Italy $>$.

INTERNATIONAL INTELLECTUAL PROPERTY ALLIANCE (IIPA). 2007. Special Report 301: Italy. <http://www.iipa.com/ rbc/2007/2007SPEC301ITALY.pdf $>$.

— 2008. Report: Italy. <http://www.iipa. com/rbc/2008/2008SPEC301ITALY.pdf $>$. 
MICOSSI, S. 2006. L'impresa tra dirigismo e mercato. Rivista di Politica Economica, jul. y ago. Págs. 36-52.

MINISTERO DEL TESORO, BILANCIO E PROGRAMMAZIONE ECONOMICA. 2003. Italy's Report on Economic Reform. Roma: Governo d'Italia.

ORGANISATION FOR ECONOMIC COOPERATION AND DEVELOPMENT (OECD). 2007. Economic Survey Italy. París: OECD.

2008a. The Economic Impact of Conterfeiting. París: OECD.

2008b. Sectoral regulatory reforms in Italy: Framework and implications. Economic Department. Working papers n. ${ }^{\circ} 294$. $<\mathrm{http}: / /$ www.oecd.org/dataoecd/32/5/1892008. pdf $>$.

PADOVANI, F. 2008. Il Potere de la TV in Italia, Il Graco, Roma.
ROMANO, R. y FERRARI, S. 2004. Privatizzazioni in Italia, L'enesima occasione mancata, Il Mulino, Roma.

THE HERITAGE FOUNDATION AND WALL STREET JOURNAL. 2008. Index of Economic Freedom. <http://europa.eu.int/comm/ dgs/olaf/index.htm>.

UNIVERSITÀ CARLO CATTANEO. 2008. The Italian elections: issues, dimensions and political positions of political parties. LIUC, Castellanza (VA).

VAN SICLEN, Sally. 2000. Italy - Regulatory Reform in Electricity, Gas and Railroads 2000. OECD's Division for Competition Law and Policy. París: OECD.

ZIMERMANN, J. 2008. Liberalisierung verändert italienischen Pharmarkt Bundesagentur für Außenwirtschaft. Institut für Wirtschaftstheorie I. Berlin: Humboldt University. 